SFB

Nonparametric tests for tail 823 monotonicity

Betina Berghaus, Axel Bücher

Nr. 7/2013
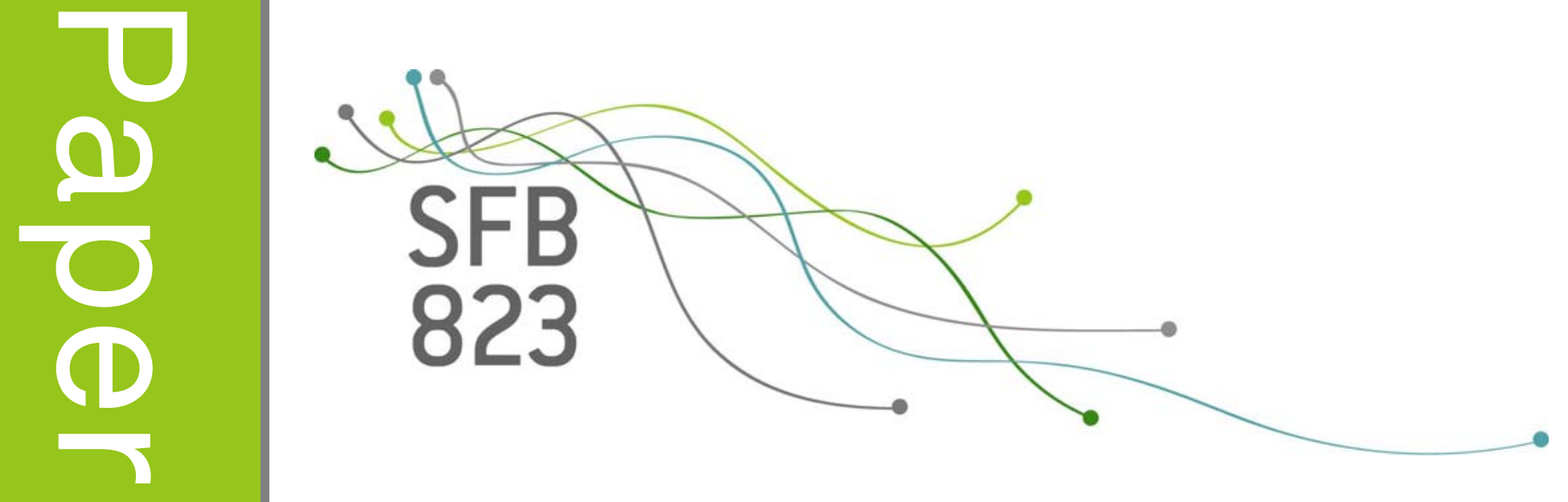



\title{
Nonparametric tests for tail monotonicity
}

\author{
Betina Berghaus and Axel Bücher \\ Ruhr-Universität Bochum \\ Fakultät für Mathematik \\ 44780 Bochum, Germany \\ e-mail: betina.berghaus@ruhr-uni-bochum.de \\ e-mail: axel.buecher@ruhr-uni-bochum.de
}

March 7, 2013

\begin{abstract}
This article proposes nonparametric tests for tail monotonicity of bivariate random vectors. The test statistic is based on a Kolmogorov-Smirnov-type functional of the empirical copula. Depending on the serial dependence features of the data, we propose two multiplier bootstrap techniques to approximate the critical values. We show that the test is able to detect local alternatives converging to the null hypothesis at rate $n^{-1 / 2}$ with a non-trivial power. A simulation study is performed to investigate the finite-sample performance and finally the procedure is illustrated by testing intergenerational income mobility.
\end{abstract}

Keywords and Phrases: Copula, Left tail decreasing, Multiplier bootstrap, Ranks, Tail monotonicity

AMS Subject Classification: Primary 62G10 ; secondary 62H15

\section{Introduction}

Let $X$ and $Y$ be random variables. The following concepts of tail monotonicity between $X$ and $Y$ date back to Lehmann (1966) and Esary and Proschan (1972). We say that

- $Y$ is left tail decreasing in $X$ if the function $x \mapsto \operatorname{Pr}(Y \leq y \mid X \leq x)$ is non-increasing for all $y \in \mathbb{R}$. Notation: $\operatorname{LTD}(Y \mid X)$.

- $Y$ is right tail increasing in $X$ if the function $x \mapsto \operatorname{Pr}(Y>y \mid X>x)$ is a non-decreasing function for all $y \in \mathbb{R}$. Notation: $\operatorname{RTI}(Y \mid X)$. 
It is well-known that all these concepts imply positive quadrant-dependence of $X$ and $Y$, i.e., $\operatorname{LTD}(Y \mid X), \operatorname{LTD}(X \mid Y), \operatorname{RTI}(Y \mid X)$ or $\operatorname{RTI}(X \mid Y)$ implies that

$$
\operatorname{Pr}(X \leq x, Y \leq y) \geq \operatorname{Pr}(X \leq x) \operatorname{Pr}(Y \leq y) .
$$

For example, under $\operatorname{LTD}(Y \mid X)$, we have $\operatorname{Pr}(Y \leq y \mid X \leq x) \geq \operatorname{Pr}(Y \leq y \mid X \leq \infty)=$ $\operatorname{Pr}(Y \leq y)$. In other words, tail monotonicity between $X$ and $Y$ as defined above implies positive dependence between $X$ and $Y$ and similar negative dependence properties known as left tail increasingness and right tail decreasingness are defined analogously by exchanging the words non-increasing and non-decreasing.

On the other hand, tail monotonicity is weaker than regression dependence or stochastic monotonicity, which is defined analogously to tail monotonicity by replacing the condition $X \leq x$ by $X=x$ hence yielding

- $Y$ is positively regression dependent on $X$ if the function $x \mapsto \operatorname{Pr}(Y \leq y \mid X=x)$ is non-increasing for all $y \in \mathbb{R}$.

While testing for the latter hypothesis, i.e., that $Y$ is positively regression dependent on $X$, or testing for positive quadrant dependence has recently drawn some attention in the literature, see Delgado and Escanciano (2012); Lee et al. (2009); Denuit and Scaillet (2004); Scaillet (2005); Gijbels et al. (2010), there does not exist an omnibus test for the hypothesis of any of the tail monotonicity concepts defined above, at least to the best of our knowledge. It is the purpose of this paper to fill this gap.

Testing for tail monotonicity is interesting for several reasons. First of all, the hypothesis can be of interest in several applied settings. As for instance summarized in Lee et al. (2009), one might wish to test for tail monotonicity in well-known economic relationships such as expenditures $(Y)$ versus income $(X)$ at household levels or wages $(Y)$ versus cognitive skills $(X)$ using individual data.

Additionally, there are some theoretical reasons which demand for a test for tail monotonicty: Genest and Segers (2010) showed that, under both $\operatorname{LTD}(Y \mid X)$ and $\operatorname{LTD}(X \mid Y)$, estimation of the copula of $(X, Y)$ by means of the empirical copula is more efficient if contingent additional knowledge of the marginal distribution function is completely ignored. This surprising result transfers to several attached statistics like the sample version of Spearman's rho. Hence, a positive testing result on the question of tail decreasingness may result in a more efficient subsequent data-analysis.

Finally, our research is motivated by a recent paper by Kojadinovic and Yan (2012). These authors propose two nonparametric tests of exchangeability of a random vector which only work for left tail decreasing random vectors. Hence, prescience of left tail decreasingness is essential for their approach.

For the sake of a clear exposition we only consider the concept of left tail decreasingness in this paper. We propose a simple nonparametric test for the hypothesis

$$
H_{0}: Y \text { is left tail decreasing in } X \text {. }
$$


The construction of variants for the increasing case or for the right tail is straight-forward.

For continuous random variables, the property $\operatorname{LTD}(Y \mid X)$ is closely related to some shape constraint on the copula of $(X, Y)$. To see this, let $F$ and $G$ denote the (continuous) cumulative distribution function (cdf) of $X$ and $Y$, respectively. Then Sklar's Theorem, see Sklar (1959), allows to decompose the joint cdf $H(x, y)=\operatorname{Pr}(X \leq x, Y \leq y)$ into

$$
H(x, y)=C\{F(x), G(y)\}
$$

where $C(u, v)=\operatorname{Pr}\{F(X) \leq u, G(Y) \leq v\}$ denotes the unique copula associated with $H$. Sklar's identity is usually interpreted in the way that the dependence structure between $X$ and $Y$ is completely summarized by $C$. Due to the fact that univariate distribution functions are non-decreasing we immediately obtain that $Y$ is left tail decreasing in $X$ if and only if

$$
H_{0}: u \mapsto C(u, v) / u=\operatorname{Pr}(G(Y) \leq v \mid F(X) \leq u)
$$

is a non-increasing function for each $v \in[0,1]$.

Set $\Delta=\left\{(s, t) \in[0,1]^{2} \mid s \leq t\right\}$ and denote by $\mathcal{T}: \ell^{\infty}\left([0,1]^{2}\right) \rightarrow \ell^{\infty}([0,1] \times \Delta)$ the operator which maps a function $H:[0,1]^{2} \rightarrow \mathbb{R}$ to the function $\mathcal{T}(H):[0,1] \times \Delta \rightarrow \mathbb{R}$ defined by

$$
\mathcal{T}(H)(u, s, t)=s H(t, u)-t H(s, u) .
$$

Here, $\ell^{\infty}(T)$ is defined as the set of all real-valued bounded functions on a set $T$. Now, nonincreasingness of the function in (1.1) is obviously equivalent to the fact that

$$
H_{0}: \mathcal{T}(C)(u, s, t) \leq 0 \text { for all } u \in[0,1],(s, t) \in \Delta .
$$

This is the property we test for in this paper. Our approach is based on the fact that the copula $C$, and therefore also $\mathcal{T}(C)$, can be estimated by its sample analog $C_{n}$, the empirical copula. We check if a suitable functional of $\mathcal{T}\left(C_{n}\right)$ is sufficiently small in a certain way. Basing the test on the empirical copula results in invariance of the test with respect to strictly increasing transformations of the marginals which should be a minimal requirement for any test of monotonicity.

Some of the afore-mentioned tests on regression dependence and positive quadrant dependence are based on the assumption of i.i.d. data sets (e.g. Delgado and Escanciano (2012); Scaillet (2005); Gijbels et al. (2010)). Exploiting a recent block multiplier bootstrap method, see Bücher and Ruppert (2012), our proposed test on tail monotonicity goes beyond this and can also be applied to serially dependent, strongly mixing data sets. By doing this, we also outline a direction on how to adopt other tests to the serially dependent setting, as long as they are based on the empirical copula.

The remaining part of this article is organized as follows. In Section 2 we define the test for tail monotonicity and propose two multiplier bootstrap procedures for deriving critical values. We begin with the easier setting of serial independent data sets and proceed with a (block) multiplier bootstrap to deal with data sets descending from a strictly stationary, strongly mixing time series. In Section 3 we investigate the finite-sample performance of the test by means of a simulation study. The approach is illustrated by testing intergenerational income mobility in Section 4, and finally, all proofs are deferred to Section 5. 


\section{Testing tail monotonicity}

Suppose $\left(X_{1}, Y_{1}\right), \ldots,\left(X_{n}, Y_{n}\right)$ is a sample of a strictly stationary stochastic process $\left(X_{i}, Y_{i}\right)_{i \in \mathbb{Z}}$ with continuous marginal cumulative distribution functions $F$ and $G$, respectively. According to Sklar's Theorem there exists a unique copula $C$ such that the joint cdf $H$ of $\left(X_{i}, Y_{i}\right)$ satisfies $H(x, y)=C(F(x), G(y))$ for all $x, y \in \mathbb{R}$. Moreover, suppose that the process $\left(X_{i}, Y_{i}\right)_{i \in \mathbb{Z}}$ is strongly mixing with $\alpha$-mixing coefficient $\alpha(r)=O\left(r^{-a}\right)$ for $r \rightarrow \infty$ and some $a>1$, where

$$
\alpha(r)=\sup _{s \geq 0} \sup _{A \in \mathcal{F}_{s}, B \in \mathcal{F}^{s+r}}|\operatorname{Pr}(A \cap B)-\operatorname{Pr}(A) \operatorname{Pr}(B)|
$$

and $\mathcal{F}_{s}=\sigma\left\{\left(X_{i}, Y_{i}\right): i \leq s\right\}$ and $\mathcal{F}^{t}=\sigma\left\{\left(X_{i}, Y_{i}\right): i \geq t\right\}$. Note that for an i.i.d. sequence $\left(X_{i}, Y_{i}\right)_{i \in \mathbb{Z}}$ we have $\alpha(r)=0$ for all $r \geq 1$.

To estimate the copula $C$ we first transform the random variables $X_{i}$ and $Y_{i}$ to pseudoobservations of the copula by defining

$$
\hat{U}_{i}=\frac{n}{n+1} F_{n}\left(X_{i}\right), \quad \hat{V}_{i}=\frac{n}{n+1} G_{n}\left(Y_{i}\right),
$$

where $F_{n}(x)=n^{-1} \sum_{i=1}^{n} \mathbf{1}\left(X_{i} \leq x\right)$ and $G_{n}(y)=n^{-1} \sum_{i=1}^{n} \mathbf{1}\left(Y_{i} \leq y\right)$ denote the sample analogs of $F$ and $G$, respectively. The empirical copula dating back to Rüschendorf (1976); Deheuvels (1979) as the most-natural nonparametric estimator for $C$ is defined as

$$
C_{n}(u, v)=\frac{1}{n} \sum_{i=1}^{n} \mathbf{1}\left(\hat{U}_{i} \leq u, \hat{V}_{i} \leq v\right) .
$$

The asymptotic behavior of the corresponding empirical copula process $\mathbb{C}_{n}=\sqrt{n}\left(C_{n}-C\right)$ has been investigated, under slightly different assumptions, by Rüschendorf (1976); Gaenssler and Stute (1987); Fermanian et al. (2004); Doukhan et al. (2009) among others. The weakest smoothness assumption on $C$ which is necessary for weak convergence of $\mathbb{C}_{n}$ was given by Segers (2012).

Condition 2.1. The copula $C$ possesses continuous first-order partial derivatives $C^{[1]}(u, v)=$ $\frac{\partial}{\partial u} C(u, v)$ and $C^{[2]}(u, v)=\frac{\partial}{\partial v} C(u, v)$ on the sets $U_{1}=(0,1) \times[0,1]$ and $U_{2}=[0,1] \times(0,1)$, respectively.

Bücher and Volgushev (2011) transferred the result from Segers (2012), who worked in the i.i.d. case, to the case of a serially dependent sample. Under Condition 2.1 and under suitable assumptions on the mixing rate, i.e., on the speed of convergence of $\alpha(r)$ to 0 , $\mathbb{C}_{n}$ weakly converges in the space $\left(\ell^{\infty}\left([0,1]^{2}\right),\|\cdot\|_{\infty}\right)$ to a process $\mathbb{C}_{C}$. Defining $C^{[1]}(u, v)=0$ on $\{0,1\} \times[0,1]$ (resp. $C^{[2]}(u, v)=0$ on $\left.[0,1] \times\{0,1\}\right)$, the limiting field $\mathbb{C}_{C}$ can be expressed as

$$
\mathbb{C}_{C}(u, v)=\mathbb{B}_{C}(u, v)-C^{[1]}(u, v) \mathbb{B}_{C}(u, 1)-C^{[2]}(u, v) \mathbb{B}_{C}(1, v),
$$

where $\mathbb{B}_{C}$ is a tight centered Gaussian process on $[0,1]^{2}$ with covariance function 


$$
\begin{aligned}
r\left((u, v),\left(u^{\prime}, v^{\prime}\right)\right)=\operatorname{Cov}\left\{\mathbb{B}_{C}(u, v), \mathbb{B}_{C}\left(u^{\prime}, v^{\prime}\right)\right\} & \\
& =\sum_{i \in \mathbb{Z}} \operatorname{Cov}\left\{\mathbf{1}\left(U_{0} \leq u, V_{0} \leq v\right), \mathbf{1}\left(U_{i} \leq u^{\prime}, V_{i} \leq v^{\prime}\right)\right\}
\end{aligned}
$$

and where $U_{i}=F\left(X_{i}\right)$ and $V_{i}=G\left(Y_{i}\right)$. Note that for serially uncorrelated time series this expression reduces to $C\left(u \wedge u^{\prime}, v \wedge v^{\prime}\right)-C(u, v) C\left(u^{\prime}, v^{\prime}\right)$, with $\wedge$ denoting the minimum between two numbers.

Omnibus tests for $H_{0}$ can be based on suitable functionals of the process

$$
\sqrt{n} \mathcal{T}\left(C_{n}\right)(u, s, t)=\sqrt{n}\left\{s C_{n}(t, u)-t C_{n}(s, u)\right\}, \quad u \in[0,1],(s, t) \in \Delta .
$$

Under $H_{0}$ this process should take small values for all $u \in[0,1]$ and $(s, t) \in \Delta$. Hence, we propose using the uniform norm, i.e., a Kolmogorov-Smirnov type criteria, and define the test statistic

$$
\tau_{n}=\sup _{(u, s, t) \in[0,1] \times \Delta} \sqrt{n} \mathcal{T}\left(C_{n}\right) .
$$

Note that other choices are possible; for instance, one could use the statistic

$$
\tilde{\tau}_{n}=\int_{[0,1] \times \Delta} n \max \left\{\mathcal{T}\left(C_{n}\right)(u, s, t), 0\right\}^{2} d(u, s, t) .
$$

It can be easily seen that

$$
\tau_{n}=\max _{1 \leq k \leq n} \max _{1 \leq i \leq n} \max _{1 \leq j \leq i} n^{-1 / 2}\left\{j C_{n}(i / n, k / n)-i C_{n}(j / n, k / n)\right\}+O\left(n^{-1 / 2}\right)
$$

Hence, for the computation of $\tau_{n}$ we only need the elements $C_{n}(i / n, j / n)$ for $i, j=1, \ldots, n$. Their computation is straightforward and can be done recursively.

We propose to test for $H_{0}$ by rejecting the null hypothesis for large values of $\tau_{n}$ and for that purpose we need to derive critical values for $\tau_{n}$. In the remaining part of this section we suggest a multiplier bootstrap based method to approximate these critical values. We prove that the resulting test is consistent and asymptotically holds the significance level, both for serially independent as well as for serial dependent data sets.

\subsection{Critical values for $\tau_{n}$ for serial independent data}

In this section we restrict ourselves to the simple case of a serially independent sample, i.e., to the i.i.d. setting. The basic idea of our proposal to obtain a consistent and asymptotically level- $\alpha$ test is as follows: linearity of $\mathcal{T}$ implies that the process $\sqrt{n}\left\{\mathcal{T}\left(C_{n}\right)-\mathcal{T}(C)\right\}$ weakly converges to $\mathcal{T}\left(\mathbb{C}_{C}\right)$, whose distribution depends in a complicated way on $C$. If we knew the $(1-\alpha)$-quantile, say $q_{C, \alpha}$, of the associated distribution of

$$
\tau_{C}:=\sup _{(u, s, t) \in[0,1] \times \Delta} \mathcal{T}\left(\mathbb{C}_{C}\right)(u, s, t)
$$


and if we rejected $H_{0}$ for $\tau_{n}>q_{C, \alpha}$, then we would clearly have

$$
\begin{aligned}
\operatorname{Pr}\left(\tau_{n}>q_{C, \alpha}\right) & \leq \operatorname{Pr}\left(\sup _{(u, s, t) \in[0,1] \times \Delta} \sqrt{n}\left\{\mathcal{T}\left(C_{n}\right)(u, s, t)-\mathcal{T}(C)(u, s, t)\right\}>q_{C, \alpha}\right) \\
& \rightarrow \operatorname{Pr}\left(\sup _{(u, s, t) \in[0,1] \times \Delta} \mathcal{T}\left(\mathbb{C}_{C}\right)(u, s, t)>q_{C, \alpha}\right)=\alpha
\end{aligned}
$$

under the null hypothesis by the Portmanteau Theorem. On the other hand, under the alternative $\tau_{n}$ would converge to infinity in probability resulting in consistency of the test. Hence, the only problem consists of getting access to the quantiles $q_{C, \alpha}$.

For that purpose we use the multiplier bootstrap technique invented by Rémillard and Scaillet (2009) in the i.i.d. time series case and further investigated in Bücher and Dette (2010). Let $\left(\xi_{i}\right)_{i=1, \ldots, n}$ be an independent sequence of random variables satisfying $\mathbb{E}\left[\xi_{i}\right]=\operatorname{Var}\left(\xi_{i}\right)=1$ for all $i=1, \ldots, n$ and let $\bar{\xi}_{n}$ denote the sample mean of $\xi_{1}, \ldots, \xi_{n}$. Define the process $\hat{\mathbb{B}}_{n}$ by

$$
\hat{\mathbb{B}}_{n}(u, v)=\sqrt{n}\left\{\frac{1}{n} \sum_{i=1}^{n} \frac{\xi_{i}}{\bar{\xi}_{n}} \mathbf{1}\left(\hat{U}_{i} \leq u, \hat{V}_{i} \leq v\right)-C_{n}(u, v)\right\}, \quad(u, v) \in[0,1]^{2} .
$$

From Theorem 2.3 in Bücher et al. (2012) it follows that $\hat{\mathbb{B}}_{n}$ weakly converges to $\mathbb{B}_{C}$, conditional on the data in probability (see Remark 5.1 for details on that type of convergence). Note that an unconditional version of this and of the following results can be found in Rémillard and Scaillet (2009) and Segers (2012). Let $h=h_{n} \rightarrow 0$ be a bandwidth such that $\inf _{n \in \mathbb{N}} h_{n} \sqrt{n}>0$ and set

$$
\begin{gathered}
C_{n}^{[1]}(u, v)= \begin{cases}\frac{C_{n}(u+h, v)-C_{n}(u-h, v)}{2 h} & \text { for all }(u, v) \in[h, 1-h] \times[0,1] \\
\frac{C_{n}(2 h, v)}{2 h} & \text { for all }(u, v) \in[0, h) \times[0,1] \\
\frac{C_{n}(1, v)-C_{n}(1-2 h, v)}{2 h} & \text { for all }(u, v) \in(1-h, 1] \times[0,1]\end{cases} \\
C_{n}^{[2]}(u, v)= \begin{cases}\frac{C_{n}(u, v+h)-C_{n}(u, v-h)}{2 h} & \text { for all }(u, v) \in[0,1] \times[h, 1-h] \\
\frac{C_{n}(u, 2 h)}{2 h} & \text { for all }(u, v) \in[0,1] \times[0, h) \\
\frac{C_{n}(u, 1)-C_{n}(u, 1-2 h)}{2 h} & \text { for all }(u, v) \in[0,1] \times(1-h, 1]\end{cases}
\end{gathered}
$$

It follows that $\hat{\mathbb{C}}_{n}$ weakly converges to $\mathbb{C}_{C}$, conditional on the data in probability, where

$$
\hat{\mathbb{C}}_{n}(u, v)=\hat{\mathbb{B}}_{n}(u, v)-C_{n}^{[1]}(u, v) \hat{\mathbb{B}}_{n}(u, 1)-C_{n}^{[2]}(u, v) \hat{\mathbb{B}}_{n}(1, v),
$$

see Bücher et al. (2012); Bücher and Ruppert (2012). Continuity of the sup-norm implies that

$$
\hat{\tau}_{n}=\sup _{(u, s, t) \in[0,1] \times \Delta} \mathcal{T}\left(\hat{\mathbb{C}}_{n}\right)(u, s, t)
$$

weakly converges to $\tau_{C}$, conditionally. Let $\hat{q}_{n, \alpha}$ denote the $(1-\alpha)$-quantile of the conditional distribution of $\hat{\tau}_{n}$. The following Theorem establishes that a test which rejects $H_{0}$ for $\tau_{n}>\hat{q}_{n, \alpha}$ is a consistent test which asymptotically holds the significance level. 
Theorem 2.2. Suppose that $\left(X_{i}, Y_{i}\right)_{i=1, \ldots, n}$ is an i.i.d. sequence of random vectors with continuous marginal distribution functions $F$ and $G$ and whose copula $C$ satisfies Condition 2.1. Then

$$
\limsup _{n \rightarrow \infty} \operatorname{Pr}\left(\tau_{n}>\hat{q}_{n, \alpha}\right) \leq \alpha \quad \text { under } H_{0}
$$

whereas

$$
\lim _{n \rightarrow \infty} \operatorname{Pr}\left(\tau_{n}>\hat{q}_{n, \alpha}\right)=1 \quad \text { under } H_{1}
$$

In practice, we get approximate access to the quantiles $\hat{q}_{n, \alpha}$ as follows: let $B$ be some reasonable large integer and for each $b=1, \ldots, B$ simulate a sample $\xi_{1}^{(b)}, \ldots, \xi_{n}^{(b)}$ as described above. For each $b$ calculate the statistic $\hat{\tau}_{n}^{(b)}$ following the indicated steps. Finally, use the the $(1-\alpha)$-sample quantile of $\hat{\tau}_{n}^{(1)}, \ldots, \hat{\tau}_{n}^{(B)}$ as an approximation for $\hat{q}_{n, \alpha}$.

Remark 2.3. In the context of testing for the similar hypothesis of quadrant dependence, Gijbels et al. (2010) proposed to approximate critical values of their corresponding test statistic by simulating from the least favorable copula under $H_{0}$. In our context the least favorable copula, i.e., the copula which is closest to the alternative, is that of a constant function $u \mapsto C(u, v) / u$ for all $v \in[0,1]$. In that case, there exists some constant $\kappa_{v}$ such that $C(u, v)=\kappa_{v} u$ and due to the fact that $C(1, v)=v$ we obtain $\kappa_{v}=v$. Hence, $C(u, v)=\Pi(u, v)=u v$ is least favorable in which case we immediately calculate that $\mathcal{T}(\Pi) \equiv 0$. The latter fact, together with the reasoning at the beginning of Section 2.1 implies weak convergence of $\tau_{n}=\tau_{n}(\Pi)$ under $C=\Pi$ to the limiting variable

$$
\tau_{\Pi}=\sup _{u \in[0,1](s, t) \in \Delta} s \mathbb{C}_{\Pi}(t, u)-t \mathbb{C}_{\Pi}(s, u) .
$$

It seems reasonable to use the quantiles of $\tau_{\Pi}$ as critical values for the test, which can be approximated by Monte Carlo simulation as accurately as desired. Unfortunately, we were not able to prove that this method results in a test which asymptotically holds the significance level. Difficulties arise from the fact there do not exist sufficiently strong results on the stochastic ordering of random variables that are suprema of Gaussian processes, such as

$$
\tau_{C}=\sup _{u \in[0,1](s, t) \in \Delta} s \mathbb{C}_{C}(t, u)-t \mathbb{C}_{C}(s, u) .
$$

For that reason, we do not follow this approach in the present paper. Moreover, note that the method would not be adoptable to the case to of serial dependent data sets as considered in the subsequent Section 2.2.

\subsubsection{Local alternatives}

As $n \rightarrow \infty$, the power of any consistent testing procedure converges to 1 under any fixed alternative. In this paragraph we show that our proposed test is able to detect local alternatives converging to the null hypothesis at rate $n^{-1 / 2}$ with a non-trivial power. To this end, we consider 
copulas which depend on the sample size defined as

$$
C^{(n)}(u, v)=\Pi(u, v)+\min \left\{\frac{\delta}{\sqrt{n}}, 1\right\} \times\{C(u, v)-\Pi(u, v)\},
$$

where $C$ is a copula that is not $\operatorname{LTD}(Y \mid X)$ and where $\delta>0$ is a constant. This copula converges to the independence copula for $n \rightarrow \infty$, but on the other hand we have

$$
\lim _{n \rightarrow \infty} \sqrt{n} \sup _{[0,1] \times \Delta} \mathcal{T}\left(C^{(n)}\right)=\delta \sup _{[0,1] \times \Delta} \mathcal{T}(C)>0 .
$$

Let $\left(X_{i, n}, Y_{i, n}\right)_{i=1, \ldots, n}$ be a triangular array of row-wise i.i.d. random vectors with continuous marginal cdf's $F^{(n)}$ and $G^{(n)}$, respectively, and with copula $C^{(n)}$. To derive the asymptotic distribution of the associated empirical copula process it is necessary to suppose that the law $P_{n}$ of $\left(F^{(n)}\left(X_{i, n}\right), G^{(n)}\left(Y_{i, n}\right)\right)_{i=1, \ldots, n}$ is contiguous with respect to the law $Q_{n}$ induced by an i.i.d. sample of size $n$ from the independence copula. More precisely, we need to suppose that for any sequence $A_{n}$ of measurable sets, $Q_{n}\left(A_{n}\right) \rightarrow 0$ as $n \rightarrow \infty$ implies $P_{n}\left(A_{n}\right) \rightarrow 0$ as $n \rightarrow \infty$. A general criterion for a sequence of probability measures to be contiguous with respect to another is given in Van der Vaart and Wellner (1996). Berg and Quessy (2009) transferred this criterion to our empirical copula setting which results in the following condition on the copula density $c$.

Condition 2.4. The copula $C$ in (2.4) is absolutely continuous with square-integrable density $c$ and

$$
\lim _{n \rightarrow \infty} \int_{0}^{1} \int_{0}^{1}\left[\sqrt{n}\left(\sqrt{c^{(n)}(u, v)}-1\right)-\frac{\delta\{c(u, v)-1\}}{2}\right]^{2} \mathrm{~d} u \mathrm{~d} v=0
$$

where $c^{(n)}(u, v)=1+\frac{\delta}{\sqrt{n}}\{c(u, v)-1\}$ denotes the density of the copula $C^{(n)}$.

For fixed $\eta>0$, let $M_{\eta}$ denote the set of all pairs $(C, \delta)$, where $C$ is a copula and $\delta>0$ is a constant, that satisfy Condition 2.4 with $\delta \sup _{[0,1] \times \Delta} \mathcal{T}(C) \geq \eta$. Note that this set is non-empty for any $\eta>0$, because every copula with bounded density fulfills Condition 2.4 for any $\delta>0$. The following theorem states that the test is able to detect local alternatives converging to the null hypothesis at rate $n^{-1 / 2}$.

Theorem 2.5. For any $\beta \in(0,1)$ there exist $\eta>0$ such that, for any sequence of local alternatives $C^{(n)}$ as defined in (2.4) with $(C, \delta) \in M_{\eta}$,

$$
\liminf _{n \rightarrow \infty} \operatorname{Pr}\left(\tau_{n}>\hat{q}_{n, \alpha}\right) \geq \beta
$$

\subsection{Critical values for $\tau_{n}$ for serial dependent data}

In this section we propose an adaptation of the methodology in the previous section which allows for the simulation of valid critical values if we drop the assumption of serial independence of the time series $\left(X_{i}, Y_{i}\right)_{i=1, \ldots, n}$. Note that the i.i.d. multiplier approach of the previous section 
would not work in this setting since it cannot take care of the serial dependence features of the time series. Therefore we make use of a refinement of this technique, called the tapered block multiplier bootstrap, which has been recently proposed by Bücher and Ruppert (2012) and which is based on earlier work by Bühlmann (1993) and Paparoditis and Politis (2001). Analogously to the i.i.d. setting, this method allows to obtain approximate samples of $\mathbb{C}_{C}$ and therefore of $\tau_{C}$ under suitable alpha-mixing conditions on the strictly stationary time series $\left(X_{i}, Y_{i}\right)_{i=1, \ldots, n}$.

The basic idea is to replace the i.i.d. multipliers $\xi_{1}, \ldots, \xi_{n}$ in the definition of $\hat{\mathbb{B}}_{n}$ in $(2.2)$ by appropriate dependent block multipliers $\xi_{1, n}, \ldots, \xi_{n, n}$. We follow the approach of Example 4 in Bücher and Ruppert (2012) which was found to give the most reasonable approximations in that reference. Precisely, let $l=l_{n} \rightarrow \infty$ be a sequence of integer-valued parameters (the block length) and for $h \in \mathbb{Z}$ denote by

$$
\kappa(h)=\max \left\{0,\left(1-|h| / l_{n}\right) / l_{n}\right\}
$$

a Kernel-function which is symmetric around 0 and satisfies $\sum_{h \in \mathbb{Z}} \kappa(h)=1$. Let $\left(w_{i}\right)_{i \in \mathbb{Z}}$ denote an i.i.d. sequence of $\operatorname{Gamma}(q, q)$ distributed random variables, where $q=2 / 3 l_{n}^{-1}+1 / 3 l_{n}^{-3}$, and define $\xi_{i, n}$ as

$$
\xi_{i, n}=\sum_{h \in \mathbb{Z}} \kappa(h) w_{i+n}, \quad i=1, \ldots, n .
$$

It follows that $\left(\xi_{i, n}\right)_{i=1, \ldots, n}$ is a $\left(2 l_{n}\right)$-dependent sequence of random variables satisfying $\mathbb{E}\left[\xi_{i, n}\right]=$ $\operatorname{Var}\left(\xi_{i, n}\right)=1$ for all $i=1, \ldots, n$, see also Section 6.2 in Bühlmann (1993). Let $\bar{\xi}_{n}$ denote the sample mean of $\xi_{1, n}, \ldots, \xi_{n, n}$ and define the process $\hat{\mathbb{B}}_{n}$ as in $(2.2)$ with $\xi_{i}$ replaced by $\xi_{i, n}$. Under suitable assumptions on the mixing rate and on the choice of $l_{n}$ it follows from the results in Bücher and Ruppert (2012) that $\hat{\mathbb{B}}_{n}$ weakly converges to $\mathbb{B}_{C}$, conditional on the data. The precise assumptions are given in Theorem 2.2 below.

Similar as in Section 2.1 and following Bücher and Ruppert (2012) we conclude that $\hat{\mathbb{C}}_{n}$ weakly converges to $\mathbb{C}_{C}$, conditional on the data, where $\hat{\mathbb{C}}_{n}$ is defined in the same way as in the case of serial independent data, see (2.3). As before, continuity of the sup-norm implies that $\hat{\tau}_{n}=\sup _{[0,1] \times \Delta} \mathcal{T}\left(\hat{\mathbb{C}}_{n}\right)$ weakly converges to $\tau_{C}$, conditionally on the data. Let $\hat{q}_{n, \alpha}$ again denote the $(1-\alpha)$-quantile of the conditional distribution of $\hat{\tau}_{n}$. The following Theorem, which is an analogue of Theorem 2.2, establishes that a test which rejects $H_{0}$ for $\hat{\tau}_{n}>\hat{q}_{n, \alpha}$ is consistent and asymptotically holds the significance level.

Theorem 2.6. Let the process $\hat{\mathbb{C}}_{n}$ be constructed as described above with block length $l_{n}=$ $O\left(n^{1 / 2-\varepsilon}\right)$ for some $\varepsilon \in(0,1 / 2)$. Suppose $F$ and $G$ are continuous and that the copula $C$ satisfies Condition 2.1. Additionally, assume that the alpha-mixing coefficients satisfy $\sum_{r=1}^{\infty}(r+$ $1)^{c} \sqrt{\alpha(r)}<\infty$, where $c=\max \{8 d+12,\lfloor 2 / \varepsilon\rfloor+1\}$. Then

$$
\limsup _{n \rightarrow \infty} \operatorname{Pr}\left(\tau_{n}>\hat{q}_{n, \alpha}\right) \leq \alpha \quad \text { under } H_{0},
$$

whereas

$$
\lim _{n \rightarrow \infty} \operatorname{Pr}\left(\tau_{n}>\hat{q}_{n, \alpha}\right)=1 \quad \text { under } H_{1} .
$$


In practice, the approximation of the quantiles $\hat{q}_{n, \alpha}$ can be done by the same procedure as in the case of serial independence by repeating the procedure reasonably often and using the corresponding sample quantiles.

\section{Remark 2.7.}

a) According to the remark following Theorem 2 in Bücher and Ruppert (2012) the conditions on the mixing rate are far from being optimal. It is beyond the scope of the present paper to improve upon general results on the block multiplier method though. Note however that popular models like ARMA- or GARCH-type processes have alpha-mixing coefficients that decay exponentially fast, hence satisfying the conditions of Theorem 2.6 with much to spare.

b) The construction of the multiplier random variables described above stems from one specific example in Bücher and Ruppert (2012) which was found to yield very reasonable results in that reference. Of course, there are different possible choices which we do not investigate in this paper.

\section{Finite-sample performance}

This section is devoted to a simulation study in order to investigate the finite-sample performance of the proposed tests for left tail decreasingness. To this end, we will simulate the rejection rates of the test under the null hypothesis and the alternative, both for serially independent and dependent data sets. The data generating processes are as followed.

- In the i.i.d. case, we simulate the rejection rates under the null hypothesis for the GumbelHougaard, the independence and the Gaussian copula, whereas under the alternative we use the Plackett and again the Gaussian copula.

- In the case of serially dependent data sets, we consider the class of strictly stationary Markovian copula models as introduced in Rémillard et al. (2012). Within this class, the 4-dimensional copula $Q$ of the vector $\left(X_{t-1}, X_{t}, Y_{t-1}, Y_{t}\right)$ is modeled in such a way that the bivariate marginal copulas $Q(u, v, 1,1)$ and $Q(1,1, u, v)$ of $\left(X_{t-1}, Y_{t-1}\right)$ and $\left(X_{t}, Y_{t}\right)$, respectively, are equal, i.e., $C(u, v)=Q(u, v, 1,1)=Q(1,1, u, v)$ is constant over time. Rémillard et al. (2012) propose algorithms that allow for the simulation of samples from Markovian copula models both for meta-elliptical copulas and for Archimedean copulas. In the latter case of Archimedean copulas, the required conditions on the corresponding generator enforce the copula to be left tail decreasing. For this reason we cannot simulate non-LTD copulas within this class, whence we restrict ourselves to the case of the Gaussian copula lying in the class of meta-elliptical copulas.

Under the null hypothesis we choose the parameters of the copula in such a way that Spearman's rho varies in the set $\{0,0.05,0.1,0.2\}$, both for serially independent as well as for serially dependent data sets. Under the alternative we choose Spearman's rho varying in the set $\{-0.05,-0.1,-0.2,-0.4\}$. For each scenario described above, we simulated 1000 samples of 


\begin{tabular}{cccccc}
\hline Copula & $\rho$ & $n=50$ & $n=100$ & $n=200$ & $n=500$ \\
\hline Gumbel & 0.2 & 0.001 & 0 & 0 & 0 \\
& 0.1 & 0.020 & 0.005 & 0.001 & 0.001 \\
& 0.05 & 0.041 & 0.033 & 0.009 & 0.001 \\
Independence & 0 & 0.109 & 0.079 & 0.050 & 0.033 \\
Plackett & -0.05 & 0.165 & 0.143 & 0.129 & 0.189 \\
& -0.1 & 0.196 & 0.279 & 0.252 & 0.417 \\
& -0.2 & 0.414 & 0.547 & 0.673 & 0.940 \\
& -0.4 & 0.987 & 0.999 & 1 & 1 \\
\hline
\end{tabular}

Table 1: Simulated rejection probabilities of the test for the null hypothesis of a copula that is LTD for serially indepentent data, where the level of the test is $5 \%$.

size $n \in\{50,100,200,500\}$. Regarding the choice of the parameters $l_{n}$ and $h_{n}$ in the bootstrap procedure we follow Bücher and Ruppert (2012) and set $l_{n}=\left\lfloor 1.1 n^{1 / 4}\right\rfloor$ and $h_{n}=n^{-1 / 4}$.

The results for independent data samples are stated in Table 1 (Gumbel-Hougaard and Plackett copula) and Figure 1 (Gaussian copula), whereas the results for dependent data samples are presented in Figure 2. The main findings are as follows.

- In the least-favorable model under the null hypothesis, i.e., the copula being equal to the independence copula, the test adapts its level. If we stay under the null hypothesis, but move away from independence, the test is globally conservative. This can be explained by the fact that only for the independence copula we have equality in (2.1). Note that similar observations have been made in Delgado and Escanciano (2012) for testing for stochastic monotonicity.

- Concerning the power, the test detects alternatives with reasonable rejection rates, where the power increases with the sample size and stronger dependence of the random variables, as expected.

- In terms of power, the test provides better results for serially independent data samples, whereas the approximation of the nominal level seems to be better for the serially dependent case.

\section{Illustration}

In this section, we apply the test for left tail decreasingness to a data set from the Panel Study of Income Dynamics (PSID), in particular the data extract presented in Minicozzi (2003), that is available on the Journal of Applied Econometrics website. The sample-size of this data set is $n=616$. Lee et al. (2009) and Delgado and Escanciano (2012) use this data set to test for 


\begin{tabular}{ccccc}
\hline$\rho$ & $n=50$ & $n=100$ & $n=200$ & $n=500$ \\
\hline 0.2 & 0.006 & 0 & 0 & 0 \\
0.1 & 0.027 & 0.013 & 0.002 & 0 \\
0.05 & 0.057 & 0.020 & 0.007 & 0 \\
0 & 0.109 & 0.079 & 0.050 & 0.033 \\
-0.05 & 0.190 & 0.185 & 0.164 & 0.243 \\
-0.1 & 0.293 & 0.355 & 0.386 & 0.681 \\
-0.2 & 0.594 & 0.767 & 0.888 & 0.998 \\
-0.4 & 0.958 & 0.999 & 1 & 1 \\
\hline
\end{tabular}

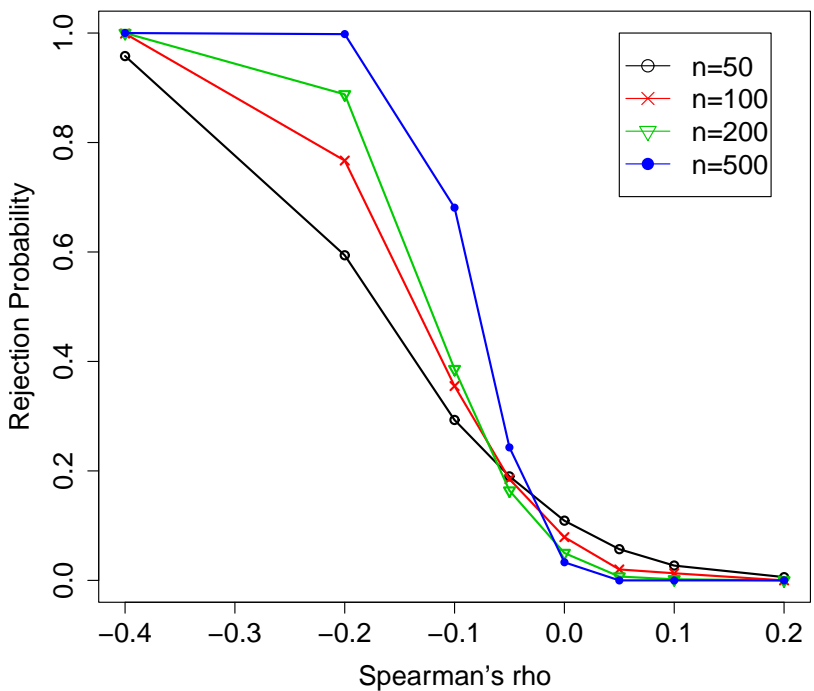

Figure 1: Simulated rejection probabilities of the test for the Gaussian copula for serially independent data, where the level of the test is $5 \%$.

\begin{tabular}{ccccc}
\hline$\rho$ & $n=50$ & $n=100$ & $n=200$ & $n=500$ \\
\hline 0.2 & 0.013 & 0.010 & 0.001 & 0 \\
0.1 & 0.035 & 0.026 & 0.005 & 0.001 \\
0.05 & 0.065 & 0.063 & 0.020 & 0.008 \\
0 & 0.064 & 0.081 & 0.049 & 0.052 \\
-0.05 & 0.136 & 0.168 & 0.111 & 0.145 \\
-0.1 & 0.188 & 0.225 & 0.189 & 0.371 \\
-0.2 & 0.341 & 0.477 & 0.530 & 0.816 \\
-0.4 & 0.824 & 0.912 & 0.933 & 0.998 \\
\hline
\end{tabular}

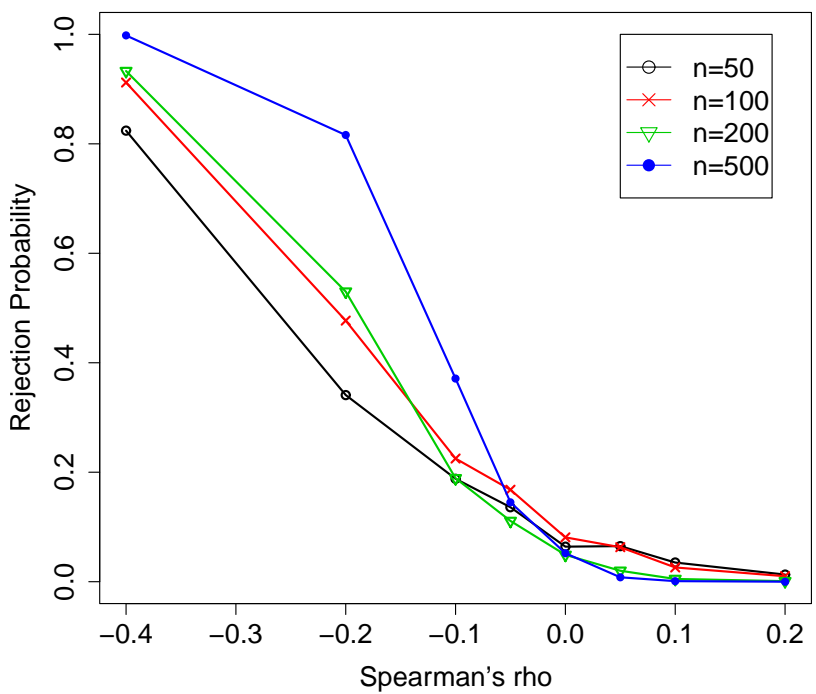

Figure 2: Simulated rejection probabilities of the test for the Gaussian copula for serially dependent data, where the level of the test is $5 \%$.

stochastic monotonicity of the variables $Y$ being the logarithm of sons' averaged full-time real labor income at ages 28 and 29 and $X$ being the logarithm of parental predicted permanent income. Both tests weren't able to reject the hypothesis of stochastic monotonicity.

We applied our test for the hypothesis that $Y$ is LTD in $X$ to the same data set and obtained a test statistic of $\tau_{n}=0.38$. For the levels $\alpha=0.05$ and 0.1 the bootstrap procedure yields 
the critical values 0.93 and 0.80 , respectively. Thus, we fail to reject the hypothesis of tail monotonicity between sons' and parental income, i.e., the hypothesis that a higher parental income implies a higher sons' income in the sense of the definition of left tail decreasingness. This is in line with the findings of Lee et al. (2009) and Delgado and Escanciano (2012), since stochastic monotonicity would imply tail monotonicity.

Furthermore, we test for tail monotonicity when the random variable $Y$ represents mothers' predicted permanent income. At first, we test with $X$ being the parental predicted permanent income and obtained a test statistic of $\tau_{n}=0.64$, which corresponds to the p-value 0.19 and thus, we cannot reject the hypothesis of tail monotonicity in this case. However, if $X$ represents the fathers' predicted permanent income, the test yields a test statistic of $\tau_{n}=1.27$. As in this case the maximum of the bootstrap test statistics $\tau_{n}^{(1)}, \ldots, \tau_{n}^{(500)}$ attains the value $1.03<1.27=\tau_{n}$, we reject the hypothesis of tail monotonicity for mothers' and fathers' predicted permanent income.

\section{Proofs}

Remark 5.1. Weak convergence conditional on the data in probability is to be understood in the Hoffmann-Jørgensen-sense, see Kosorok (2008). More precisely, for some (possibly nonmeasurable) map $\hat{\alpha}_{n}$ taking values in some metric space $\mathbb{D}$ and being dependent on both the data $\left(X_{1}, Y_{1}\right), \ldots,\left(X_{n}, Y_{n}\right)$ and on the multipliers $\xi_{1}, \ldots, \xi_{n}$, we use the notation $\hat{\alpha}_{n} \underset{\xi}{\stackrel{\mathbb{P}}{\xi}} \alpha$ to mean that

$$
\sup _{h \in B L_{1}(\mathbb{D})}\left|\mathbb{E}_{\xi} h\left(\hat{\alpha}_{n}\right)-\mathbb{E} h(\alpha)\right| \stackrel{\mathbb{P}}{\rightarrow} 0
$$

and

$$
\mathbb{E}_{\xi} h\left(\hat{\alpha}_{n}\right)^{\star}-\mathbb{E}_{\xi} h\left(\hat{\alpha}_{n}\right)_{\star} \stackrel{\mathbb{P}}{\rightarrow} 0 \quad \text { for each } h \in B L_{1}(\mathbb{D}) .
$$

Here, $B L_{1}(\mathbb{D})$ denotes the set of all Lipschitz-continuous functions $h: \mathbb{D} \rightarrow \mathbb{R}$ that are bounded by1 with Lipschitz-constant not exceeding 1 . Moreover, $\mathbb{E}_{\xi}$ denotes the conditional expectation over the multipliers given the data and $h\left(\hat{\alpha}_{n}\right)^{\star}$ and $h\left(\hat{\alpha}_{n}\right)_{\star}$ denote measurable majorants and minorants with respect to the joint data, including the multipliers.

Proof of Theorem 2.2. Let us first prove that the test asymptotically holds the significance level. For copulas $C$ that are left tail decreasing and satisfy the assumptions of the Theorem, the argumentation preceding (2.1) shows that

$$
\limsup _{n \rightarrow \infty} \operatorname{Pr}\left(\tau_{n}>q_{C, \alpha}\right) \leq \alpha .
$$

Hence, it suffices to show that

$$
\lim _{n \rightarrow \infty}\left|\operatorname{Pr}\left(\tau_{n}>\hat{q}_{n, \alpha}\right)-\operatorname{Pr}\left(\tau_{n}>q_{C, \alpha}\right)\right|=0
$$


From the fact that $\hat{\mathbb{C}}_{n}$ weakly converges to $\mathbb{C}_{C}$ in $\ell^{\infty}\left([0,1]^{2}\right)$, conditional on the data in probability, and from the continuous mapping theorem for the bootstrap, see Proposition 10.7 in Kosorok (2008), we obtain

$$
\hat{\tau}_{n}=\sup _{(s, t, u) \in \Delta \times[0,1]} s \hat{\mathbb{C}}_{n}(t, u)-t \hat{\mathbb{C}}_{n}(s, u) \underset{\xi}{\stackrel{\mathbb{P}}{\xi}} \sup _{(s, t, u) \in \Delta \times[0,1]} s \mathbb{C}_{C}(t, u)-t \mathbb{C}_{C}(s, u)=\tau_{C} .
$$

As a consequence of Lemma 10.11 in Kosorok (2008) and the results in (van der Vaart, 1998, p. 329 ) we see that the conditional quantile $\hat{q}_{n, \alpha}$ converges in probability to the quantile $\hat{q}_{C, \alpha}$. Due to the fact that $\tau_{C}$ is a continuous random variable this implies $\left|\operatorname{Pr}\left(\tau_{C}>\hat{q}_{n, \alpha}\right)-\operatorname{Pr}\left(\tau_{C}>q_{C, \alpha}\right)\right| \rightarrow 0$. Finally, the estimation

$$
\begin{aligned}
& \left|\operatorname{Pr}\left(\tau_{n}>\hat{q}_{n, \alpha}\right)-\operatorname{Pr}\left(\tau_{n}>q_{C, \alpha}\right)\right| \\
\leq & \left|\operatorname{Pr}\left(\tau_{n}>\hat{q}_{n, \alpha}\right)-\operatorname{Pr}\left(\tau_{C}>\hat{q}_{n, \alpha}\right)\right| \\
& \quad+\left|\operatorname{Pr}\left(\tau_{C}>\hat{q}_{n, \alpha}\right)-\operatorname{Pr}\left(\tau_{C}>q_{C, \alpha}\right)\right|+\left|\operatorname{Pr}\left(\tau_{C}>q_{C, \alpha}\right)-\operatorname{Pr}\left(\tau_{n}>q_{C, \alpha}\right)\right| \\
\leq & 2 \sup _{x \in \mathbb{R}}\left|\operatorname{Pr}\left(\tau_{n} \leq x\right)-\operatorname{Pr}\left(\tau_{C} \leq x\right)\right|+o(1)
\end{aligned}
$$

proves the assertion about the level of the test. Under the alternative we first notice that there exists at least one $\left(u_{0}, s_{0}, t_{0}\right) \in[0,1] \times \Delta$ with $\mathcal{T}(C)\left(u_{0}, s_{0}, t_{0}\right)>0$. Without loss of generality we may assume that $\left(X_{i}, Y_{i}\right) \sim C$, i.e., that $F(x)=G(x)=x$ for all $x \in[0,1]$. Defining $\tilde{C}_{n}(u, v)=n^{-1} \sum_{i=1}^{n} \mathbf{1}\left\{X_{i} \leq F_{n}^{-1}(u), Y_{i} \leq G_{n}^{-1}(v)\right\}$, we have

$$
\begin{aligned}
\left\|C_{n}-C\right\|_{\infty} \leq\left\|C_{n}-\tilde{C}_{n}\right\|_{\infty}+\sup _{(u, v) \in[0,1]^{2}} \mid \tilde{C}_{n}(u, v) & -C\left(F_{n}^{-1}(u), G_{n}^{-1}(v)\right) \mid \\
& +\sup _{(u, v) \in[0,1]^{2}}\left|C\left(F_{n}^{-1}(u), G_{n}^{-1}(v)\right)-C(u, v)\right| .
\end{aligned}
$$

The first summand on the right-hand side is bounded by $2 n^{-1}$. By the Glivenko-Cantelli Theorem the second summand converges to 0 almost surely. Finally, uniform continuity of $C$ and $\| F_{n}^{-1}-$ $F \|_{\infty}=o(1)$ almost surely, implies that $\left\|C_{n}-C\right\|_{\infty} \rightarrow 0$, almost surely. Therefore, the estimation

$$
\tau_{n} \geq \sqrt{n} \mathcal{T}\left(C_{n}\right)\left(u_{0}, s_{0}, t_{0}\right)=\sqrt{n} \mathcal{T}(C)\left(u_{0}, s_{0}, t_{0}\right)+o(\sqrt{n}), \text { a.s. }
$$

shows that $\tau_{n}$ converges to infinity in probability. The fact that $\hat{\mathbb{B}}_{n}=O_{P}(1)$ and hence $\hat{\mathbb{C}}_{n}=$ $O_{P}(1)$ and $\hat{q}_{n, \alpha}=O_{P}(1)$ implies consistency of the test.

Proof of Theorem 2.5. Following Berg and Quessy (2009) and Van der Vaart and Wellner (1996) we have

$$
\mathbb{C}_{n}^{(n)}=\sqrt{n}\left(C_{n}-\Pi\right) \rightsquigarrow \mathbb{C}_{\Pi}+\delta(C-\Pi),
$$

which implies

$$
\tau_{n} \rightsquigarrow \sup _{[0,1] \times \Delta}\left(\mathcal{T}\left(\mathbb{C}_{\Pi}\right)+\delta \mathcal{T}(C)\right) .
$$


Let us verify that the conditional quantiles $\hat{q}_{n, \alpha}$ converge to $q_{\Pi, \alpha}$ in probability. Define unobservable random variables $\left(U_{i, n}, V_{i, n}\right)=\left(F^{(n)}\left(X_{i, n}\right), G^{(n)}\left(Y_{i, n}\right)\right) \sim C^{(n)}$ and set

$$
\tilde{\mathbb{B}}_{n}(u, v)=\sqrt{n}\left\{\frac{1}{n} \sum_{i=1}^{n} \frac{\xi_{i, n}}{\bar{\xi}_{n}} \mathbf{1}\left(U_{i, n} \leq u, V_{i, n} \leq v\right)-C_{n}(u, v)\right\}, \quad(u, v) \in[0,1]^{2} .
$$

By Theorem 11.13 in Kosorok (2008) we have $\tilde{\mathbb{B}}_{n} \rightsquigarrow \mathbb{B}_{\Pi}$, conditional on the data in probability. The same proof as the one of Theorem 2.3 in Bücher et al. (2012) shows that also $\hat{\mathbb{B}}_{n} \rightsquigarrow \mathbb{B}_{\Pi}$, conditional on the data in probability. In order to show conditional weak convergence of $\hat{\mathbb{C}}_{n}$ to $\mathbb{C}_{\Pi}$, let us first prove that, for any $\delta \in(0,1 / 2)$ and $j=1,2$,

$$
\sup _{\left(u_{1}, u_{2}\right) \in B_{j}^{\delta}}\left|C_{n}^{[j]}\left(u_{1}, u_{2}\right)-\Pi^{[j]}\left(u_{1}, u_{2}\right)\right|=o_{p}(1)
$$

as $n \rightarrow \infty$, where $B_{j}^{\delta}=\left\{\left(u_{1}, u_{2}\right) \in[0,1]^{2}: u_{j} \in[\delta, 1-\delta]\right\}$. To this end, we only consider $j=1$ and estimate

$$
\begin{aligned}
\sup _{\left(u_{1}, u_{2}\right) \in B_{1}^{\delta}} \mid C_{n}^{[1]}\left(u_{1}, u_{2}\right)- & \Pi^{[1]}\left(u_{1}, u_{2}\right) \mid \\
\leq & \sup _{\left(u_{1}, u_{2}\right) \in B_{1}^{\delta}}\left|\frac{\Pi\left(u_{1}+h_{n}, u_{2}\right)-\Pi\left(u_{1}-h_{n}, u_{2}\right)}{2 h_{n}}-\Pi^{[1]}(\mathbf{u})\right| \\
& +\frac{1}{2 h_{n} \sqrt{n}} \sup _{\left(u_{1}, u_{2}\right) \in B_{1}^{\delta}}\left|\mathbb{C}_{n}^{(n)}\left(u_{1}+h_{n}, u_{2}\right)-\mathbb{C}_{n}^{(n)}\left(u_{1}-h_{n}, u_{2}\right)\right| .
\end{aligned}
$$

The first term on the right of the previous inequality is equal to zero. Under consideration of $\inf _{n \in \mathbb{N}} h_{n} \sqrt{n}>0$, asymptotic uniform equicontinuity of $\mathbb{C}_{n}^{(n)}$ implies that the second term converges to zero in probability. This proves (5.1).

Now, a similar argumentation as in the proof of Theorem 2.5 in Bücher et al. (2012) and an application of Lemma B.1 in that reference allows to conclude that $\hat{\mathbb{C}}_{n} \rightsquigarrow \mathbb{C}_{\Pi}$ and hence $\hat{\tau}_{n} \rightsquigarrow \tau_{\Pi}$, conditional on the data in probability. As in the proof of Theorem 2.2 this implies convergence of $\hat{q}_{n, \alpha}$ to $q_{\Pi, \alpha}$ in probability. Therefore, again by the same arguments as in the proof of Theorem 2.2 , it is sufficient to prove (2.5) with $\hat{q}_{n, \alpha}$ replaced by $q_{\Pi, \alpha}$.

Now, due to the fact that $\mathcal{T}(C):[0,1] \times \Delta \rightarrow \mathbb{R}$ is continuous, we may choose an $\left(u_{0}, s_{0}, t_{0}\right) \in$ $[0,1] \times \Delta$ such that $\mathcal{T}(C)\left(u_{0}, s_{0}, t_{0}\right)=\sup _{[0,1] \times \Delta} \mathcal{T}(C)>0$. This implies

$$
\sup _{[0,1] \times \Delta}\left(\delta \mathcal{T}(C)+\mathcal{T}\left(\mathbb{C}_{\Pi}\right)\right) \geq \delta \mathcal{T}(C)\left(u_{0}, s_{0}, t_{0}\right)+\mathcal{T}\left(\mathbb{C}_{\Pi}\right)\left(u_{0}, s_{0}, t_{0}\right)
$$

Hence, for any $\varepsilon>0$ we can find $n_{0}$ large enough such that

$$
\begin{aligned}
\operatorname{Pr}\left(\tau_{n}>q_{\Pi, \alpha}\right) & \geq \operatorname{Pr}\left(\sup _{[0,1] \times \Delta}\left(\delta \mathcal{T}(C)+\mathcal{T}\left(\mathbb{C}_{\Pi}\right)\right)>q_{\Pi, \alpha}\right)-\varepsilon \\
& \geq \operatorname{Pr}\left(\delta \mathcal{T}(C)\left(u_{0}, s_{0}, t_{0}\right)+\mathcal{T}\left(\mathbb{C}_{\Pi}\right)\left(u_{0}, s_{0}, t_{0}\right)>q_{\Pi, \alpha}\right)-\varepsilon
\end{aligned}
$$


for all $n \geq n_{0}$. Moreover, for any $\gamma \in(0,1)$ we can choose $\eta$ large enough such that, for any $(C, \delta) \in M_{\eta}$, we have

$$
\operatorname{Pr}\left(\delta \mathcal{T}(C)\left(u_{0}, s_{0}, t_{0}\right)+\mathcal{T}\left(\mathbb{C}_{\Pi}\right)\left(s_{0}, t_{0}, u_{0}\right)>q_{\Pi, \alpha}\right) \geq \operatorname{Pr}\left(\eta+\mathcal{T}\left(\mathbb{C}_{\Pi}\right)\left(u_{0}, s_{0}, t_{0}\right)>q_{\Pi, \alpha}\right)>\gamma .
$$

Finally, set $\gamma=(\beta+1) / 2$ and $\varepsilon=(1-\beta) / 2$ in the latter two inequalities to finish the proof.

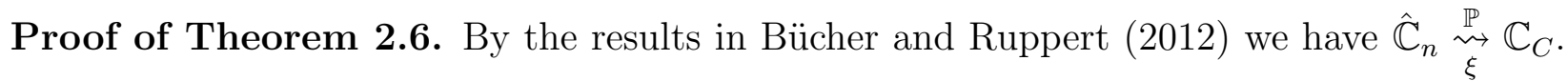
Hence, the assertion follows by the same arguments as in the proof of Theorem 2.2. The details are omitted for the sake of brevity.

Acknowledgements. This work has been supported by the Collaborative Research Center "Statistical modeling of nonlinear dynamic processes" (SFB 823) of the German Research Foundation (DFG).

\section{References}

Berg, D. and Quessy, J.-F. (2009). Local power analyses of goodness-of-fit tests for copulas. Scand. J. Stat., 36(3):389-412.

Bücher, A. and Dette, H. (2010). A note on bootstrap approximations for the empirical copula process. Statist. Probab. Lett., 80:1925-1932.

Bücher, A., Dette, H., and Volgushev, S. (2012). A test for Archimedeanity in bivariate copula models. J. Multivariate Anal., 110:121-132.

Bücher, A. and Ruppert, M. (2012). Consistent testing for a constant copula under strong mixing based on the tapered block multiplier technique. arXiv:1206.1675. submitted for publication.

Bücher, A. and Volgushev, S. (2011). Empirical and sequential empirical copula processes under serial dependence. arXiv:1111.27r8.

Bühlmann, P. L. (1993). The blockwise bootstrap in time series and empirical processes. ProQuest LLC, Ann Arbor, MI. Thesis (Dr.Sc.Math)-Eidgenoessische Technische Hochschule Zürich (Switzerland).

Deheuvels, P. (1979). La fonction de dépendance empirique et ses propriétés. Un test non paramétrique d’indépendance. Acad. Roy. Belg. Bull. Cl. Sci. (5), 65(6):274-292.

Delgado, M. A. and Escanciano, J. C. (2012). Distribution-free tests of stochastic monotonicity. Journal of Econometrics, 170(1):68 - 75 .

Denuit, M. and Scaillet, O. (2004). Nonparametric tests for positive quadrant dependence. Journal of Financial Econometrics, 2(3):422-450. 
Doukhan, P., Fermanian, J.-D., and Lang, G. (2009). An empirical central limit theorem with applications to copulas under weak dependence. Stat. Inference Stoch. Process., 12(1):65-87.

Esary, J. D. and Proschan, F. (1972). Relationships among some concepts of bivariate dependence. Ann. Math. Statist., 43:651-655.

Fermanian, J.-D., Radulović, D., and Wegkamp, M. (2004). Weak convergence of empirical copula processes. Bernoulli, 10(5):847-860.

Gaenssler, P. and Stute, W. (1987). Seminar on empirical processes, volume 9 of DMV Seminar. Birkhäuser Verlag, Basel.

Genest, C. and Segers, J. (2010). On the covariance of the asymptotic empirical copula process. J. Multivariate Anal., 101(8):1837-1845.

Gijbels, I., Omelka, M., and Sznajder, D. (2010). Positive quadrant dependence tests for copulas. Canadian Journal of Statistics, 38(4):555-581.

Kojadinovic, I. and Yan, J. (2012). A non-parametric test of exchangeability for extreme-value and left-tail decreasing bivariate copulas. Scandinavian Journal of Statistics, 39(3):480-496.

Kosorok, M. R. (2008). Introduction to empirical processes and semiparametric inference. Springer Series in Statistics. Springer, New York.

Lee, S., Linton, O., and Whang, Y.-J. (2009). Testing for stochastic monotonicity. Econometrica, $77(2): 585-602$.

Lehmann, E. L. (1966). Some concepts of dependence. Ann. Math. Statist., 37:1137-1153.

Minicozzi, A. (2003). Estimation of sons' intergenerational earnings mobility in the presence of censoring. Journal of Applied Econometrics, 18(3):291-314.

Paparoditis, E. and Politis, D. N. (2001). Tapered block bootstrap. Biometrika, 88(4):1105-1119.

Rémillard, B., Papageorgiou, N., and Soustra, F. (2012). Copula-based semiparametric models for multivariate time series. J. Multivariate Anal., 110:30-42.

Rémillard, B. and Scaillet, O. (2009). Testing for equality between two copulas. J. Multivariate Anal., 100(3):377-386.

Rüschendorf, L. (1976). Asymptotic distributions of multivariate rank order statistics. Annals of Statistics, 4:912-923.

Scaillet, O. (2005). A Kolmogorov-Smirnov type test for positive quadrant dependence. Canad. J. Statist., 33(3):415-427. 
Segers, J. (2012). Asymptotics of empirical copula processes under non-restrictive smoothness assumptions. Bernoulli, 18(3):764-782.

Sklar, A. (1959). Fonctions de répartition à $n$ dimensions et leurs marges. Publ. Inst. Statist. Univ. Paris, 8:229-231.

van der Vaart, A. W. (1998). Asymptotic statistics, volume 3 of Cambridge Series in Statistical and Probabilistic Mathematics. Cambridge University Press, Cambridge.

Van der Vaart, A. W. and Wellner, J. A. (1996). Weak Convergence and Empirical Processes Springer Series in Statistics. Springer, New York. 

